\title{
Confirmation of factors that influence antiretroviral regimen change and the subsequent patient outcomes at a Regional Hospital in rural KwaZulu-Natal
}

\begin{tabular}{|c|c|}
\hline $\begin{array}{l}\text { Authors: } \\
\text { Vereesha Soo } \\
\text { Panjasaram N }\end{array}$ & $\begin{array}{l}\text { rju }^{1} \\
\text { aidoo }^{1}\end{array}$ \\
\hline $\begin{array}{l}\text { Affiliations: } \\
{ }^{1} \text { Madadeni Hc } \\
\text { of Health Scie } \\
\text { of KwaZulu-N } \\
\text { South Africa }\end{array}$ & $\begin{array}{l}\text { spital, School } \\
\text { nces, University } \\
\text { atal, }\end{array}$ \\
\hline $\begin{array}{l}\text { Research Proj } \\
\text { BE 206/15 }\end{array}$ & ject no.: \\
\hline $\begin{array}{l}\text { Correspondin } \\
\text { Vereesha Soo } \\
\text { soorjuv@gma }\end{array}$ & $\begin{array}{l}\text { g author: } \\
\text { rju, } \\
\text { il.com }\end{array}$ \\
\hline $\begin{array}{l}\text { Dates: } \\
\text { Received: } 21 \\
\text { Accepted: } 28 \\
\text { Published: } 31\end{array}$ & $\begin{array}{l}\text { Feb. } 2016 \\
\text { July } 2016 \\
\text { Oct. } 2016\end{array}$ \\
\hline $\begin{array}{l}\text { How to cite th } \\
\text { Soorju V, Naid } \\
\text { Confirmation } \\
\text { influence anti } \\
\text { regimen chan } \\
\text { subsequent } p \\
\text { at a Regional } \\
\text { KwaZulu-Nata } \\
\text { Health Care F } \\
2016 ; 8(1) \text {, a1 } \\
\text { dx.doi.org/10 } \\
\text { v8i1.1171 }\end{array}$ & $\begin{array}{l}\text { his article: } \\
\text { loo P. } \\
\text { of factors that } \\
\text { retroviral } \\
\text { ge and the } \\
\text { atient outcomes } \\
\text { Hospital in rural } \\
\text { l. Afr J Prm } \\
\text { am Med. } \\
\text { 171. http:// } \\
.4102 / \text { phcfm. }\end{array}$ \\
\hline $\begin{array}{l}\text { Copyright: } \\
\text { ( ) 2016. The } \\
\text { Licensee: AOS } \\
\text { is licensed un } \\
\text { Creative Com } \\
\text { Attribution Lic }\end{array}$ & $\begin{array}{l}\text { Authors. } \\
\text { is. This work } \\
\text { der the } \\
\text { mons } \\
\text { cense. }\end{array}$ \\
\hline Read online: & \\
\hline 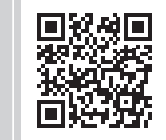 & $\begin{array}{l}\text { Scan this QR } \\
\text { code with your } \\
\text { smart phone or } \\
\text { mobile device } \\
\text { to read online. }\end{array}$ \\
\hline
\end{tabular}

Background: Treatment failure (TF) and adverse drug reactions (ADRs) are the main indications for antiretroviral therapy (ART) regimen change. Identification of factors influencing regimen change and subsequent health outcomes of patients after regimen change is essential in providing a sustainable and effective antiretroviral roll-out campaign.

Aim: To confirm the factors that influence antiretroviral regimen change and to evaluate patient outcomes post regimen change.

Methods: A retrospective chart analysis of 269 HIV-infected non-pregnant patients (age $>18$ years), who underwent an antiretroviral (ARV) regimen change and were followed up for approximately one year since initiation, was undertaken at a Provincial Hospital ARV Clinic in KwaZulu-Natal, from January 2008 to December 2012.

Results: Of the 269 patients, there were 200 females (75\%). Most patients were between the ages 30 and 44 (57.6\%). Only five patients had coexisting tuberculosis (TB) infection (2\%). The most common first-line ART regimen to be changed was stavudine (D4T)/ lamivudine(3TC)/ efavirenz(EFV) $n=111(41 \%)$. The most common regimen patients were changed to was tenofovir (TDF)/3TC/EFV $n=89(33 \%)$. Stavudine was the most commonly substituted drug (35.5\%). Lipodystrophy was the most common ADR (56.8\%). ADR was the indication for ART regimen change in 175 patients $(65 \%)$, whilst TF accounted for ART regimen change in 94 patients (35\%). Immunological success (CD4 counts) was shown after regimen change $(374.21 \pm 243.16$ vs. $456.09 \pm 250.07, C I: 0.95, p<0.001)$. Undetectable viral loads were measured in 172/205 (83.9\%) patients post regimen change.

Conclusion: ADRs were the main cause for antiretroviral regimen change. Stavudine was the most substituted drug with lipodystrophy being the most common side effect. Coexisting TB infection did not influence regimen change. Immunological and virological success was shown after regimen modification.

\section{Introduction}

South Africa has the highest number of HIV-infected people worldwide with an estimated 5.6 million people living with HIV. ${ }^{1}$ A strategic report in 2005 reported an antenatal seroprevalence of $22 \%$, which rose to $30 \%$ in 2014 reflecting an increase in the number of HIV infection in the population. ${ }^{1}$ The antiretroviral roll-out campaign in South Africa has gained much momentum in the last decade. In April 2010, treatment was offered in fewer than 500 facilities - by the end of 2013 that number had risen to over 3500, and the number of people receiving antiretroviral therapy (ART), from less than 1 million to greater than 2 million. ${ }^{1}$

Despite the success in access and distribution of ART, several challenges exist with respect to adverse drug reactions (ADRs) and treatment failure (TF), which are indications for ART regimen change. ${ }^{2}$ These two factors are intimately related as an ADR may lead to poor adherence, which ultimately results in TF. ${ }^{2}$ During the period 2007-2011, drug-related toxicity from first-line regimens was found to be a major contributing factor to ART regimen change in the South African context. ${ }^{3}$ ART regimen change can be challenging when drug availability, cost and acceptable combinations are limited by their pharmacokinetic and toxicity profiles, thus allowing for only a certain number and class of drug substitutions to occur. Personal determinants of poor adherence have been investigated, which included factors, such as stigma, discrimination, depression and alcohol, that would negatively affect patients' outcomes. $^{2}$ 
The resurgence of tuberculosis (TB) has largely paralleled the HIV pandemic with more than $50 \%$ of HIV-infected patients developing TB in their lifetime. Highly active antiretroviral therapy (HAART) has led to a decrease in HIV-associated TB by more than $80 \%{ }^{4}$ Despite the reported success, several drug interactions exist between ART and anti-TB therapy consequently affecting the pharmacokinetic properties of ART, often resulting in reduced plasma levels of both nonnucleoside reverse transcriptase inhibitors and protease inhibitors (PI). ${ }^{4,5}$ This may contribute to TF, thus influencing ART regimen change.

Frequent monitoring enables the diagnosis of virological failure before the development of drug resistance mutations, which would ultimately lead to TF and allow for possible viral transmission. ${ }^{6}$

Monitoring the immunological (CD4) and virological response (viral load[VL]) to ART regimen change is essential in determining the efficacy of regimen changes and individual drug substitutions.

Hence, the aim of this study was to confirm and quantify the factors that influence ART regimen change and to evaluate the health outcomes of the patients post regimen change using CD4 and VL as markers.

\section{Methods}

A retrospective chart analysis of 269 HIV-infected patients who underwent an ART regimen change between January 2008 and December 2012 and who were followed up for approximately one year after an ART regimen change at a rural Provincial Hospital ARV Clinic, situated in Newcastle, KwaZulu-Natal, South Africa, was undertaken. Patients who had a regimen change were sampled in chronological order. Data were manually retrieved from outpatient files.

Patients under 18 years of age and pregnant patients were excluded from the data analysis, the latter being excluded because the Madadeni Hospital ARV Clinic does not render a maternity and paediatric service. Variables included in the data analysis were age, gender, first-line ART regimen, coexisting TB infection, modified ART regimen, ADRs, drug substitutions, indication for regimen change and time to regimen change.

Outcome measures included the viral load and CD4 counts prior to ART regimen change and at six months follow up after ART regimen change. An undetectable viral load was given a numerical value of 39 for purposes of statistical analysis.

Descriptive statistics, using means and standard deviations for continuous variables, and frequencies for categorical variables, were used to report sample characteristics. A Student's $t$-test was used to compare continuous variables. A $p<0.05$ was considered statistically significant. SPSS version 23 software (Chicago, IL, USA) was used for the data analysis.

\section{Ethical considerations}

Gatekeepers permission was given by the KwaZulu-Natal Department of Health Research and Development Committee with the study given full ethical approval from the Biomedical Research Ethics Committee of the University of KwaZuluNatal (BREC No: 206/15).

\section{Results}

Of the 269 patients who underwent ART regimen change, there were 200 females (75\%) and 69 males (25\%). Most patients were between the ages 30 and $44(57 \%)$; >45 years (25\%) and $18-29$ years (16\%). Only five patients had coexisting TB infection (2\%) (Table 1).

The most common first-line ART regimens to be changed were stavudine (D4T)/lamivudine (3TC)/ efavirenz (EFV) $n=111(41 \%), \mathrm{D} 4 \mathrm{~T} / 3 \mathrm{TC} /$ nevirapine $(\mathrm{NVP}) n=100(37 \%)$ and $\mathrm{TDF} / 3 \mathrm{TC} / \mathrm{EFV} n=26(10 \%)$.

TABLE 1: Socio-demographic and immunological profile (entire cohort).

\begin{tabular}{lc}
\hline Variables & $\boldsymbol{n}(\%)$ \\
\hline Gender & $200(74.3)$ \\
Females & $69(25.7)$ \\
Males & \\
Age & $43(16)$ \\
18-29 years & $159(59.1)$ \\
$30-44$ years & $67(24.9)$ \\
$>45$ years & 34.6 \\
Time to change (months) & $5(2)$ \\
Coexisting TB infection & \\
Indication for ART change & $175(65)$ \\
Adverse drug reaction & $94(35)$ \\
Treatment failure & $374.21 \pm 243.16$ \\
Preceding CD4 count (cells/mm ${ }^{3}$ ) (mean \pm s.d.) & \\
Preceding viral load (copies/mL) & $168(62.5)$ \\
Undetectable & $5(1.9)$ \\
$40-499$ & $96(35.7)$ \\
$>1000$ &
\end{tabular}

Source: The contents of Tables 1-5 are from our data analysis from the data collected from the ARV Clinic at Madadeni Hospital

ART, antiretroviral therapy; TB, tuberculosis.

TABLE 2: Frequency of initial antiretroviral therapy (ART) regimen.

\begin{tabular}{lc}
\hline Initial ART regimen & $\boldsymbol{N}(\boldsymbol{\%})$ \\
\hline AZT/3TC/EFV & $5(1.9)$ \\
AZT/3TC/LPVr & $1(0.4)$ \\
AZT/3TC/NVP & $3(1.1)$ \\
AZT/ABC/LPVr & $1(0.4)$ \\
AZT/DDI/LPVr & $1(0.4)$ \\
D4T/3TC/EFV & $111(41.3)$ \\
D4T/3TC/LPVr & $1(0.4)$ \\
D4T/3TC/NVP & $100(37.2)$ \\
TDF/3TC/EFV & $26(9.7)$ \\
TDF/3TC/NVP & $20(7.4)$ \\
\hline
\end{tabular}

Source: The contents of Tables 1-5 are from our data analysis from the data collected from the ARV Clinic at Madadeni Hospital

ART, antiretroviral therapy; D4T, stavudine; 3TC, lamivudine; EFV, efavirenz; NVP, nevirapine; AZT, zidovudine; DDI, didanosine; TDF, tenofovir; ABC, abacavir; LPVr, lopinavir/ritonavir; $N$, number. 
TABLE 3: Frequency of modified antiretroviral therapy (ART) regimen.

\begin{tabular}{lc}
\hline Modified ART regimen & $N(\%)$ \\
\hline $\mathrm{ABC} / 3 T C / E F V$ & $1(0.4)$ \\
$\mathrm{ABC} / 3 \mathrm{TC} / \mathrm{LPVr}$ & $5(1.9)$ \\
$\mathrm{AZT} / 3 \mathrm{TC} / \mathrm{EFV}$ & $1(0.4)$ \\
$\mathrm{AZT} / 3 T C / \mathrm{LPVr}$ & $40(14.9)$ \\
$\mathrm{AZT} / 3 \mathrm{TC} / \mathrm{NVP}$ & $2(0.7)$ \\
$\mathrm{D} 4 \mathrm{~T} / 3 \mathrm{TC} / \mathrm{LPVr}$ & $1(0.4)$ \\
$\mathrm{TDF} / 3 \mathrm{TC} / \mathrm{EFV}$ & $89(33.1)$ \\
$\mathrm{TDF} / 3 \mathrm{TC} / \mathrm{LPVr}$ & $55(20.4)$ \\
$\mathrm{TDF} / 3 \mathrm{TC} / \mathrm{NVP}$ & $75(27.9)$ \\
\hline
\end{tabular}

Source: The contents of Tables 1-5 are from our data analysis from the data collected from the ARV Clinic at Madadeni Hospital

ART, antiretroviral therapy; D4T, stavudine; 3TC, lamivudine; EFV, efavirenz; NVP, nevirapine; AZT, zidovudine; DDI, didanosine; TDF, tenofovir; ABC, abacavir; LPVr, lopinavir/ritonavir; $N$, number.

TABLE 4: Frequency of adverse drug reactions in entire cohort $(n=269)$.

\begin{tabular}{lcccccc}
\hline ADRs & 3TC & AZT & D4T & EFV & NVP & Total \\
\hline Lipodystrophy & 0 & 2 & 150 & 0 & 0 & 152 \\
Anaemia & 0 & 2 & 0 & 0 & 0 & 2 \\
Peripheral neuropathy & 0 & 0 & 7 & 0 & 0 & 7 \\
Lactic acidosis & 0 & 0 & 1 & 0 & 0 & 1 \\
Skin reaction & 0 & 0 & 0 & 0 & 1 & 1 \\
Steven Johnson syndrome & 0 & 0 & 0 & 0 & 3 & 1 \\
CNS effects & 0 & 0 & 0 & 2 & 0 & 2 \\
\hline Total & $\mathbf{0}$ & $\mathbf{4}$ & $\mathbf{1 5 8}$ & $\mathbf{2}$ & $\mathbf{4}$ & $\mathbf{1 6 8}$ \\
\hline
\end{tabular}

Source: The contents of Tables 1-5 are from our data analysis from the data collected from the ARV Clinic at Madadeni Hospital

ADRs, adverse drug reactions; 3TC, lamivudine; AZT, zidovudine; D4T, stavudine; EFV, efavirenz; NVP, nevirapine; CNS, central nervous system.

TABLE 5: Outcome of ART regimen change - CD4 count data analysis between TF and ADR groups.

\begin{tabular}{lccc}
\hline Variable & $\begin{array}{c}\text { Preceding CD4 } \\
\left(\text { cells } / \mathrm{mm}^{3}\right) \\
\text { Mean } \pm \text { s.d. }\end{array}$ & $\begin{array}{c}\text { Post change CD4 } \\
\left(\text { cells } / \mathrm{mm}^{3}\right) \\
\text { Mean } \pm \text { s.d. }\end{array}$ & $\boldsymbol{p}$ \\
\hline Treatment failure & $174.28 \pm 144.37$ & $290.66 \pm 186.60$ & $p<0.001$ \\
Adverse drug reaction & $500.16 \pm 212.375$ & $543.90 \pm 233.56$ & $p=0.003$ \\
\hline Total cohort & $\mathbf{3 7 4 . 2 1} \pm 243.16$ & $\mathbf{4 5 6 . 0 9} \pm 250.07$ & $\boldsymbol{p}<0.001$ \\
\hline
\end{tabular}

Source: The contents of Tables 1-5 are from our data analysis from the data collected from the ARV Clinic at Madadeni Hospital

s.d., standard deviation.

The most common regimens that patients changed to included $\mathrm{TDF} / 3 \mathrm{TC} / \mathrm{EFV} n=89(33 \%), \mathrm{TDF} / 3 \mathrm{TC} / \mathrm{NVP} n=75(28 \%)$ and TDF $/$ NVP $/$ LPV $n=55(20 \%)$ (Tables 2 and 3). The three most common drug substitutions were D4T $n=144(35.5 \%)$, EFV $n=34(12.6 \%)$ and NVP $n=34(12.6 \%)$.

The most common ADR was lipodystrophy (56.8\%) (Table 4).

ADR was the indication for ART regimen change in 175 patients $(65 \%)$ and $\mathrm{TF}$ reason for change in 94 patients $(35 \%)$.

There was a significant difference in the time to regimen change in the TF and ADR groups (30.77 vs. 36.72 months), $p<0.002$. The mean follow up time for CD4 and viral load counts was 14.31 months.

There was a significant increase in the mean CD4 counts post regimen change in the entire cohort $(374.21 \pm 243.16$ [pre] vs. $456.09 \pm 250.07$ [post]), $p<0.001$. There was a significant difference between the CD4 counts post regimen change in the TF and ADR groups $(290.65 \pm 180.60$ vs. $543.90 \pm 233.56$ cells $/ \mathrm{mL}), p<0.001$ ) (Table 5).
Pre-ART regimen change viral load data are shown in Table 1. Sixty-four patients had missing post regimen change viral load data. Of the remaining 205 patients with post regimen change viral load data, 172/205 (83.9\%) patients had undetectable viral loads after regimen change, 40-499 copies/mL: 11(5.4\%) and >1000 copies/mL: 18(8.8\%).

\section{Discussion}

\section{Main reasons for antiretroviral therapy regimen change}

The main indication for ART regimen change globally includes TF and ADR. ${ }^{3}$ In this study, ADR (65\%) was the most frequent indication for ART regimen change. The high frequency of ADR in this study may be attributed to the high number of first-line regimens containing stavudine, which accounted for the highest number of drug-specific toxicity, namely lipodystrophy. This finding is consistent with a study done by Lima et al. who have shown that $88.5 \%$ of ART regimen changes were because of ADR requiring drug substitutions. ${ }^{7}$ In another study, Kumarasamy et al. have demonstrated that the most common reason for changing ART regimens was ADR $(64 \%),{ }^{8}$ whereas Sidavasan et al. have shown drug toxicity to account for $27 \%$ of their ART regimen changes. ${ }^{9}$ Locally, Orell et al. have shown that only $1.7 \%$ of patients changed to a second-line regimen because of drug toxicity, ${ }^{10}$ but this study was conducted between 2002 and 2005 at which time second-line regimens were not routinely available in South Africa. Isolated drug substitutions were more commonly practised at the time.

TF accounted for 35\% of ART regimen change in this study. The low prevalence of TF may be as a result of improved levels of patient adherence because of robust counselling and patient education. Reasons cited in studies for virological failure include poor adherence, genetic factors and drug interactions, which lead to reduced antiretroviral (ARV) concentrations and transmitted drug resistance. ${ }^{11}$ Locally, Dube et al. had shown that TF was the second most common reason for regimen change. ${ }^{3}$

TF has far more serious implications when switching to a second-line regimen because of the inclusion of PI. The major pitfalls of a change to a second-line PI-based regimen include complex and frequent dosing, which may lead to poor adherence.

A study conducted by Paterson et al. of patients on PI-based regimens showed that adherence was significantly associated with successful virologic outcome $(p<0.001)$ and increase in CD4 lymphocyte count $(p<0.006)$. Virologic failure was documented in $22 \%$ of patients with adherence of $95 \%$ or greater, $61 \%$ of those with $80-95 \%$ adherence, and $80 \%$ of those with less than $80 \%$ adherence. ${ }^{12}$

Coexisting tuberculosis infection and antiretroviral therapy regimen change

In this study, coexisting TB infection occurred in only $2 \%$ of the study population and was a non-contributory factor 
influencing ART regimen change. Lima et al. have shown that drug interactions between ART and anti-TB drugs accounted for only $3.8 \%$ of ART regimen changes. ${ }^{7}$

In contrast, a study by Dean et al., which involved HIV and TB co-infected patients, presented a total of 167 adverse events that were recorded in 99 (54\%) of the 183 patients for whom data on therapy were available. Adverse events led to cessation or interruption of either their TB or HIV therapy in $63(34 \%)$ of the 183 patients. The most common side effects noted were peripheral neuropathy, rash, gastrointestinal, hepatitis and neurological events. ${ }^{13}$

Dean et al. also showed that peripheral neuropathy was the most frequently documented adverse event (21\%). Twentytwo of these 39 patients (56\%) were taking ARV concomitantly. This adverse event led to an interruption of TB/HIV therapy in 19 cases. $^{9}$ Sixteen $(27 \%)$ of 56 patients who received isoniazid and stavudine concomitantly developed peripheral neuropathy compared with 21 of 124 (17\%) patients prescribed isoniazid without stavudine. ${ }^{13}$ In our study, the low prevalence of TB co-infection and the use of concomitant anti-TB therapy did not impact on drug-drug interactions with stavudine, which was the most common agent used in the entire cohort.

There are various reasons why patients may develop peripheral neuropathy (advanced HIV disease, TB, antituberculous or ART and nutritional deficiencies); the study by Dean et al. indicated that this side effect was mainly observed in patients prescribed isoniazid with antiretrovirals known to be neurotoxic. ${ }^{13}$ The study stated an observed rate that was higher than that reported in HIV-infected patients without TB who were prescribed stavudine and didanosine (8\%) and HIV-negative patients prescribed isoniazid for TB $(0.15 \%)$. However, other studies reported a greatly increased incidence of peripheral neuropathy (55\%) for HIV-infected patients prescribed isoniazid and stavudine concomitantly. ${ }^{13}$

Although the study by Dean et al. showed that a higher percentage of patients receiving this combination developed peripheral neuropathy compared with patients receiving isoniazid alone, this did not attain statistical significance. Given that a high proportion of these patients had either their TB or HIV medication changed in an attempt to alleviate the symptoms, caution should be exercised when prescribing multiple agents known to cause nerve damage in high-risk patients. ${ }^{13}$

Despite the low incidence of reported ART-anti-TB therapy drug interactions in this study, clinicians should still be aware of the potential for toxicity and clinically monitor for side effects. ${ }^{13}$

\section{Most common adverse drug reactions}

ADRs were most commonly caused by stavudine (D4T) $(35.5 \%)$ with associated lipodystrophy being the most common side effect in this study. Other studies have shown stavudine toxicity accounted for $76 \%$ of ART regimen changes. ${ }^{9}$

A local study by Dube et al. has established that $34 \%$ of patients had changed from their initial ARV regimen. This study revealed that of the patients who had changed regimen because of ARV-related toxicity, $76.1 \%$ of patients changed from a stavudine-based regimen to a zidovudine-based regimen. ${ }^{3}$

In addition, the study by Dube et al. depicted that the most common adverse effects experienced by patients were polyneuropathy (24\%), lipodystrophy (23.9\%), neuropathy $(10 \%)$ and suspected lactic acidosis $(3.8 \%){ }^{3}$

However, Lima et al. have shown that zidovudine (AZT) was the highest substituted drug (42.4\%) with anaemia as the most common adverse side effect. ${ }^{7}$ The significant toxicity associated with D4T and especially after cumulative exposure has led to the removal of D4T as part of first-line ARV treatment according to the National Department of Health $(\mathrm{NDOH})$ guidelines as of $2015 .{ }^{14}$ A fixed dose combination (FDC) of TDF/FTC/EFV has been implemented as part of first-line treatment since 2013. The benefits of phasing out of D4T include the exclusion of a significantly toxic drug, a reduction in stigma associated with lipodystrophy and lipoatrophy, and a decline in mortality because of lactic acidosis. ${ }^{14}$ Our findings have shown that $99 \%$ of patients were changed to a non-D4T-containing regimen in keeping with NDOH guidelines.

The inability of patients to manage the side effects associated with ARVs may result in poor adherence of treatment regimens. Management of HIV is a multidisciplinary approach. It is imperative to screen patients for signs and symptoms of adverse effects or drug toxicity. Pharmacists play a key role in ensuring the safe, rational and appropriate use of medicine, as well as provision of pharmaceutical care by monitoring patient outcomes.

The mean time to ART regimen change was 34.5 months with a significantly higher duration in the ADR group. This may be attributed to the high frequency of lipodystrophy in this study population, which fares as a tolerable side effect thus delaying the urgency for ART regimen change.

Although Dube et al. have indicated that the average time to regimen change was 14.9 months, ${ }^{3}$ Sivadasan et al. have shown that serious drug toxicities (skin reactions) occur as early as from 2 weeks up to 42 weeks (lactic acidosis) necessitating earlier ART regimen change. ${ }^{9}$

\section{Monitoring of outcomes of antiretroviral therapy regimen change}

The outcome of ART regimen change to a second-line regimen can be monitored clinically (WHO AIDS clinical staging), 
immunologically (CD4 counts) and virologically (viral load). We chose to monitor the VL and CD4 at 6 months post regimen change to assess the therapeutic outcome. In this study, the mean follow up CD4 and VL counts were done at 14.31 months. The significant delay may be attributed to poor socio-economic status and lack of transport infrastructure especially in the rural setting.

There was a statistically significant increase in the CD4 count following regimen change, suggesting immunological success in both TF and ADR groups. The significant increase in the post regimen change CD4 count in the ADR group may be attributed to poor patient adherence when experiencing intolerable side effects. Virological success was achieved in $83.9 \%$ of patients who had undetectable viral load after changing regimen. The WHO has proposed that $70 \%$ of patients should achieve virological suppression $(<400$ copies $)$ $\mathrm{mL}$ ) after 6 months of ART. ${ }^{15}$ Another study had shown a treatment success rate of $82 \%$ following a change to a secondline regimen at 1 year follow up. ${ }^{16}$ In our study, the mean gain in CD4 count post regimen change at 14.31 months was 82 cells $/ \mathrm{mm}^{3}$, which compares favourably with local studies from Johannesburg that reported a mean increase in CD4 count of 133 cell $/ \mathrm{mm}^{3}$ following regimen change. ${ }^{17}$ Because of poor laboratory support and follow up, viral loads were not routinely measured in $64 / 269$ patients $(24 \%)$. In a study from Khayelitsha, South Africa, Boulle et al. have shown that viral load monitoring is an essential tool in the early identification of TF and for monitoring patient adherence even in the resource constrained setting. ${ }^{18}$

Frequent monitoring enables the diagnosis of virological failure before the development of drug resistance mutations, which would ultimately lead to TF and allow for possible viral transmission. ${ }^{6}$ In the absence of virological monitoring, immunological monitoring by CD4 count change is recommended. However, CD4 testing has a poor accuracy and low positive predictive value for diagnosing TF. The limitations associated with immunological monitoring could lead to patients being diagnosed very late or misdiagnosed completely, with the result that patients can be kept on a failing regimen or switched unnecessarily; hence, routine virological monitoring remains the gold standard. ${ }^{6}$

\section{Limitations}

Incomplete sets of data, particularly the lack of viral load data in the entire cohort, precluded an accurate assessment of virological response rates following ART regimen change. The rural-based site of the study may not be a representative of the HIV-infected patient population at large, where poor socio-economic status delays to hospital care and poor follow up of CD4 and VL are more prevalent.

\section{Conclusion and recommendations}

This study has confirmed that both ADR and TF are in fact reasons for ART regimen change in patients attending this rural ARV Clinic with ADR being a more common reason for change than TF. The most commonly implicated drug was stavudine (D4T) with its associated toxicity, namely lipodystrophy being the most common side effect. TB was not a determining factor influencing ART regimen change. The change of regimen had resulted in significant improvements in both post ART regimen immunological and virological success rates. This highlights the importance of stringent patient follow up, early identification of ADR and timeous switching to an alternative regimen.

Readily available access to ARV clinics, routine monitoring of patients and appropriate patient counselling constitute key elements in ensuring favourable patient outcomes.

\section{Acknowledgements}

The authors thank Prof Glenda Matthews (statistician) for the statistical analysis, the staff of Madadeni Hospital ARV Clinic and Department of Pharmacy, Mr. NTW Mchunu and Ms. Juanita Meyer for their support.

\section{Competing interests}

The authors declare that they have no financial or personal relationships that may have inappropriately influenced them in writing this article.

\section{Authors' contributions}

V.S. developed research protocol and collected, collated and analysed the data. V.S. prepared the manuscript which was edited by P.N. P.N. supervised the entire research project.

\section{References}

1. Cerenata C. 10 years of ART in South Africa - How far have we come? S Afr J HIV Med. 2014;15(1):14-15. http://dx.doi.org/10.7196/SAJHIVMED

2. Negash T, Ehlers V. Personal factors influencing patients' adherence to ART in Addis Ababa, Ethiopia. J Assoc Nurses AIDS Care. 2013;24(6):530-538. http:// dx.doi.org/10.1016/j.jana.2012.11.004

3. Dube NM, Summers R, Tint KS, Mayayise G. A pharmacovigilance study of highly active antiretroviral therapy. Pan Afr Med J. 2012;11:39

4. Badri M, Wilson $D$, Wood R. Effect of highly active antiretroviral therapy on incidence of tuberculosis in South Africa: A cohort. Lancet. 2002;359:2059-2064. http://dx.doi.org/10.1016/s0140-6736(02)08904-3

5. de Maat MMR, Huitema ADR, Mulder JW, Meenhorst PL, van Gorp ECM, Beijnen $\mathrm{JH}$. Population pharmacokinetics of nevirapine in an unselected cohort of HIV-1infected individuals. Br J Clin Pharmacol. 2002;54(4):378-385. http://dx.doi. org/10.1046/j.1365-2125.2002.01657.x

6. Roberts T, Bygrave H, Farjardo E, Ford N. Challenges and opportunities for the implementation of virological testing in resource - Limited settings. J Int AIDS Soc. 2012;15:17324. http://dx.doi.org/10.7448/JAS.15.2.1732

7. Lima D, Arruda E, Lima A, Oliveira B, Fonteles M. Factors determining changes in antiretroviral therapy. Rev Assoc Med Bras. 2011;58(2):222-228. http://dx.doi. org/10.1016/S0104-4230(12)70184-7

8. Kumarasamy N, Vallabhaneni $\mathrm{S}$, Cecelia AJ, et al. Reasons for modification of generic highly active antiretroviral therapeutic regimens among patients in southern India. J Acquir Immune Defic Syndr. 2006;41(1):53-58. http://dx.doi. southern India. J Acquir Immune Defic Syndr.
org/10.1097/01.qai.0000188123.15493.43

9. Sivadasan $A$, Abraham OC, Rupali $P$, et al. High rates of regimen change due to drug toxicity among a cohort of South Indian adults with initiated on generic, firstline antiretroviral treatment. J Assoc Indian Phys. 2009;57(2):384-388

10. Orrell C, Harling G, Lawn SD, et al. Conservation of first line antiretroviral treatment regimen where therapeutic options are limited. Antivir Ther 2007;12(1):83-88

11. Negash T, Ehlers V. Personal factors influencing patients' adherence to ART in Addis Ababa, Ethiopia. JANAC. 2013;24(6):530-538. http://dx.doi.org/10.1016/j. jana.2012.11.004

12. Peterson DL, Swindells $S$, Mohr J, et al. Adherence to protease inhibitor therapy and outcomes in patients with HIV infection. Ann Intern Med. 2000;133:21-30. $\mathrm{http}: / / \mathrm{dx}$.doi.org/10.7326/0003-4819-133-1-200007040-00004 
13. Dean GL, Edwards SG, Ives NJ, et al. Treatment of tuberculosis in HIV-infected persons in the era of highly active antiretroviral therapy. AIDS. 2002;16:75-83. http://dx.doi.org/10.1097/00002030-200201040-00010

14. World Health Organisation. Stavudine (d4T) phase - Out management: Guiding principles [homepage on the Internet]. 2015 [cited 2015 Nov 18] Available from: http://www.who.int/HIV/topics/treatment

15. Calmy A, Ford N, Hirschel B, et al. HIV viral monitoring in resource limited regions: Optional or necessary? Clin Infect Dis. 2007;44:128-144. http://dx.doi. org/10.1086/510073

16. Patel D, Desai M, Shah AN, Dikshit RN. Early outcome of second line antiretroviral therapy in treatment experienced human immunodeficiency virus positive patients. Perspect Clin Res. 2013;4(4):215-220. http://dx.doi.org/10.4103/22293485.120170

17. Fox MP, Ive $P$, Long $L$, Maskew $M$, Sanne L. High rates of survival, immune reconstitution, and virological suppression on second line antiretroviral therapy in South Africa. J Acquired Immune Defic Syndr. 2010;53(4):500-506. http://dx. doi.org/10.1097/OAl.0b013e3181bcdac http://dx doi.org/10.4103/2229-3485. 120170

18. Boulle A, Van Cutsem G, Coetzee D, Hilderbrand K, Goemaere E, Maartens G. Regimen durability and tolerability to 36-month duration of ART in Khayelitsha, South Africa [abstract 66]. Program and abstracts of the 13th Conference on Retroviruses and Opportunistic Infections; 2006 Feb 5-8; Denver. Alexandria, VA: Foundation for Retrovirology and Human Health; 2006 